\title{
Aproximaciones conceptuales de categorías de la realidad social vinculadas a la extensión universitaria
}

\section{Conceptual approaches of categories of social reality linked to university extension \\ Priscilla Mena García}

Escuela de Planificación y Promoción Social, Universidad Nacional, Costa Rica pris.mena@gmail.com

\section{Carmen Monge Hernández}

Vicerrectoría de Extensión, Universidad Nacional, Costa Rica

carmen.monge.hernandez@una.cr

\section{Randy Chavarría Briceño \\ Escuela de Planificación y Promoción Social, Universidad Nacional, Costa Rica randy.chavarria.briceno@una.cr}

Recibido: 24/09/2019 • Aceptado: 20/05/2021

\begin{abstract}
Resumen
Latinoamérica sostiene grandes desafíos sociales y medioambientales para el desarrollo humano sostenible, donde la misión social estatutaria vinculada a la extensión universitaria recobra valor, pertinencia y prioridad en la educación superior. Actualmente, surgen importantes cuestionamientos y vacíos conceptuales para alcanzar una extensión de contenido crítico e integrador. El presente artículo muestra los resultados de un análisis conceptual, los cuales destacan claves de partida de las problemáticas socio ambientales desde el proceso de
\end{abstract}


extensión universitaria. La finalidad es reflexionar respecto a cuestionamientos sobre la realidad social conceptos como sociedad, entorno, paradigmas y el cambio social. Se concluyó que la definición de extensión se asume desde una cosmovisión que determina el posicionamiento de quienes la ejecutan respecto a las categorías que involucra -tratadas en el texto y otras posibles-, así como de la dimensión axiológica y comunicacional de la universidad ante la sociedad a la que se debe. Reflexionar desde la visión del modelo crítico e integrador de extensión implica el compromiso sociopolítico de la universidad de hacer realidad el mejoramiento profesional y humano de sus estudiantes y el ideario altruista de la nación a la que responde.

Palabras clave: extensión universitaria, cambio social, sociedad, entorno, comunidad.

\section{Abstract}

Latin America presents great social and environmental challenges to sustainable human development, in which the statutory social mission associated with university outreach gains new value, relevance and priority in higher education. Currently, important questions and conceptual gaps arise in outreach efforts which involve critical and integrative content. This article shows the results of a conceptual analysis that highlight key aspects of the socio-environmental problems related to the university outreach process. The purpose of this analysis is to reflect on questions about social reality, referring to concepts such as society, environment, paradigms and social change. It is concluded that the definition of outreach is influenced by a worldview that determines the position of those who carry it out with respect to the categories it involves, which are addressed in the text and other sources, as well as the axiological and communications-related dimensions of the university as perceived by society. Adopting the vision of the critical and integrative outreach model entails a socio-political commitment of the university to making the professional and human improvement of its students a reality, responding to the altruistic ideology of the nation which this goal reflects.

Keywords: university extension, social change, society, environment, community. 


\section{Introducción}

Latinoamérica heredó de la colonización un modelo de universidad clasista y elitista, caracterizada por profundas desigualdades en el acceso y desvinculación con los intereses de su entorno. En 1918, con la Reforma Universitaria de Córdoba en Argentina, se logró un viraje significativo en la orientación hacia la democratización de la educación superior. Los cambios conseguidos permitieron remagnificar su impronta social que hasta la fecha es la diferencia en el ámbito mundial (Monge, 2020; Monge, González \& Méndez, 2020 y Tünnermann, 2000).V Si bien esa reforma surgió en el marco del Primer Congreso Nacional de Estudiantes Universitarios, el resultado se le otorga esencialmente a la clase media que consiguió romper con el dominio del poder oligárquico y clerical en el acceso y gestión de las universidades (Tünnermann, 2000). Con la incorporación de la extensión universitaria, como una función inherente e inseparable de la sociedad (González, 1996), las universidades de la región asumieron una misión social aún vigente.

Las universidades públicas latinoamericanas son consideradas un bien público, albergan un derecho humano y, por ello, el interés de garantizarlo para todas las personas. Tienen el encargo social de justicia, de garantizar condiciones de equidad y participación en el acceso, gratuidad en los servicios y velar por eludir todo tipo de exclusiones y desigualdades sociales (Monge, González \& Méndez, 2020). Esto le confiere un rol protagónico en la democratización del conocimiento y en la calidad de sus procesos formativos y funciones sustantivas universitarias (docencia, investigación y extensión), por lo tanto, en la formación de profesionales integrales y la construcción de agendas en investigación y extensión que atiendan las demandas locales y globales no únicamente lo que solicita el mercado.

A pesar de esa orientación social, los procesos sustantivos universitarios y la gestión universitaria tienen dificultades para conseguir la comprensión de las condiciones sociales de las personas, los sentidos de lo político, las relaciones de poder, las exclusiones sociales y los requerimientos ambientales planetarios (Monge et al, 2020). Actualmente hay críticas sobre la criticidad frente a los desafíos ambientales y las amenazas del modelo económico dominante, como lo expresa Núñez, "conocemos, vivimos y sufrimos (pobreza, marginación, exclusión, deterioro ambiental etc.) [que] son consecuencias obvias del sistema general de poder hegemónico 
neoliberal" (2006, pp. 20-21). Este autor concluye que los esfuerzos de los principales actores redundan en fortalecer el poder económico, político, social y cultural en torno a dichos intereses.

Lo anterior sugiere una universidad capaz de generar no solo los conocimientos técnicos y científicos necesarios para el desarrollo del país, sino también ser motor de construcción democrática, justa y equitativa. Por lo tanto, de conocimiento no condicionado por los códigos del lucro, que reconstruya la cultura necesaria para hacer frente a los desafíos de la humanidad y del planeta. Si la universidad es considerada un elemento del mercado, no hay espacio para la crítica en los debates públicos. Al actuar como agente mediador entre actores, sectores e instituciones, se desarrolla como una acción colectiva crítica de la propia institución, tanto en su ámbito interno como en sus relaciones con la sociedad (Mollis, 2003, p. 205).

Estos desafíos ponen frente a las diversas disciplinas, como las ciencias sociales, no solo buscar comprender la realidad, sino también promover procesos y funciones sustantivas universitarias que promuevan la transformación social. Un ejemplo lo constituye la Escuela de Planificación y Promoción Social, cuyo objeto de estudio es la realidad y su transformación. Si la extensión universitaria persigue ese acercamiento a la realidad, con el diálogo de saberes que realiza la universidad con la sociedad (Ghiso, 2000), mediante sus procesos sustantivos, se destaca la comunicación como la vía expedita de transformación (Freire, 1984). Surge la necesidad, también, de determinar el modelo de extensión a que se acoge la universidad, puesto que en dependencia de ello será el carácter y naturaleza de sus propósitos y resultados de cambio social, cambio que incluye la formación profesional de sus estudiantes de pre y posgrado.

Ante los diversos retos del proceso de extensión universitaria, este artículo se propone analizar conceptualmente la extensión como proceso sustantivo universitario y también aquellas categorías implicadas en su definición, tales como: realidad social, sociedad, entorno, comunidad, paradigmas y cambio social. El trabajo se constituye inicialmente de una breve sistematización de la definición de extensión, seguido, del análisis de las categorías antes expuestas y, finalmente, se enuncian las conclusiones más relevantes en relación con el objetivo declarado. 


\section{La extensión como punta de partida}

Para iniciar el tema de las diferentes concepciones de extensión, expresada en sus definiciones se considera clave partir de los rasgos históricos y filosóficos de la extensión universitaria. Resulta fundamental entender la extensión desde su origen en la Segunda Guerra Mundial a consecuencia del desarrollo industrial, sobre todo en Europa. Posteriormente, su desarrollo en América Latina a partir de las grandes y frecuentes luchas sociales. En Europa la sociedad demandaba principalmente de la universidad en función de acceso a tecnología, en tanto Latinoamérica solicitaba apoyo en su lucha por transformaciones sociales (González, 1996). A continuación, se presentan las Ecaracterísticas de sus principales modelos y concepciones.

\section{Diferentes concepciones del término extensión}

En un contexto monástico y colonizador, la universidad, considerada élite del saber, ejecutaba la extensión interviniendo, literalmente, las comunidades; modelo que Valsagna (1997) clasifica como tradicionalista. Con el avance del desarrollo científico-técnico en el contexto capitalista surgen formas economicistas, que permanecen vigentes y se presentan con gran frecuencia. Posterior al auge de los movimientos progresistas, sobre todo en Latinoamérica, inspirado en las tendencias pedagógicas de la liberación y al enfoque sociocultural de la pedagogía Vigotstky, (1896-1934), se define el modelo integral de extensión difundido principalmente en Cuba. Este modelo aboga por el cumplimiento de principios dialógicos en la comunicación universidad-sociedad, de interactividad, contextuación y creatividad (González, 1996; González, 2002; González, 2006). Mientras que el modelo crítico (Tommasino \& Cano, 2016; Tommasino, González, Guedes \& Prieto, 2006) se evidencia en Uruguay y Argentina principalmente y, más recientemente, el modelo transformador, pensado desde el enfoque de las capacidades de desarrollo humano, aplicado al entorno desde experiencias de universidades públicas de Costa Rica, Sudáfrica y Argentina (Monge, 2020).

Cabe destacar que, entre los primeros modelos de extensión, post reforma de Córdoba, destacaron el tradicionalista, altruista y divulgativo (González, 2013), reflejos de las visiones propias de una universidad iluminista, con relaciones de carácter unidireccionales, colonizadoras e intervencionistas. 
El modelo altruista impregnó en los fines de la reforma el pensamiento positivista de la época, pero desde acciones entusiastas, desinteresadas y compasivas a favor de las personas más desposeídas de las sociedades. El divulgativo reprodujo las nociones extensionistas norteamericanas de transferir, con los medios de comunicación, los conocimientos técnicos y la cultura universitaria a las poblaciones sin acceso a la universidad. Tales modelos presentaron la característica fundamental de ser mínimos en su contenido crítico político y en el aporte a la construcción colectiva hacia la transformación social, demandada por las sociedades (Monge et al, 2020). Según Tünnermann (1981), los proyectos y actividades de esta época solían presentar las siguientescaracterísticas:

1. No daban respuesta a programas debidamente diseñados con objetivos definidos.

2. Presentaban un carácter marginal en su contribución por su poca vinculación con la investigación y la docencia.

3. Se sustentaban en una extensión orientada a la difusión cultural.

4. Daban respuesta principalmente a demandas clasistas y a propósitos informativos más que a fines concientizadores y formativos.

En la década de mil novecientos sesenta, la extensión y la educación en general, presentaron cambios de rumbo sustanciales con los aportes de los pedagogos brasileños (Monge et al, 2020). Esto se observa en los aportes centrados en trabajar el concepto y definición de la extensión universitaria. En esta época surge la extensión concientizadora que recoge las ideas provocadoras de diálogo y la comunicación; de una extensión que impugna la transmisión de la ideología dominante y, al contrario, promueve una actitud transformadora (Freire, 1965).

En el concepto extensión universitaria desde las concepciones de modelos más progresistas como el integral, crítico y transformador, el término extensión resulta perturbador y contradictorio. Ya Pablo Freire, desde la década de mil novecientos ochenta, rechazó el término extensión, pues lo relacionó con "procesos de invasión cultural, transmisión, entrega, donación, mesianismo, relaciones de dominación y opresión a los que se han visto sometidos, diferentes sectores de la sociedad" (Freire, 1984, p. 
20). Dicho autor concluyó que estos términos llevan acciones que ponen al individuo como una "cosa", y no como un ser que puede lograr transformaciones. Ante tal perspectiva, proposo el uso del término comunicación, considerando que aquel proceso se caracteriza por permitir la reciprocidad, el diálogo y, con ello, se revela y modifica la realidad que conduce a liberación. Además, la comunicación promueve que los sujetos interlocutores incidan su admiración sobre el mismo objeto, sin que se rompa la relación pensamiento-lenguaje-contexto o realidad ya que no hay pensamiento que no esté referido a la realidad $y$, por tanto, marcado por ella.

Esta última concepción sobre cómo todo está marcado o determinado por la realidad, se relaciona con lo señalado por Durkheim (1986). El autor comprende que los hechos sociales son aquellos que contribuyen a comprender la realidad al poner en manifiesto las necesidades que influyen en la sociedad. Es decir, la realidad no se puede entender desde el escritorio o de manera aislada, sino únicamente de forma totalizadora.

Bosco (1973) refuerza lo antes planteado cuando señala que la extensión puede conducir a la dominación al asociarla con la acción de extender el conocimiento a personas o colectivos. En otras palabras, advierte que la extensión puede reducirse a una simple transferencia de un sujeto que tiene el conocimiento (o cree tenerlo), a otros. De esta manera, se vería de forma iluminista y elitista a los agentes de la universidad que transfieren conocimiento a aquellos que no lo poseen. Por ende, un acto muy lejos de la relación que se requiere hoy de las universidades para con la sociedad.

Ante estos conceptos, Tommasino et al. (2006) proponen que se reconozca por una parte lo que Freire señaló como modelo extensionista clásico y, por otra parte, integra la crítica de Bosco (1973), argumentando que, si se entiende como tal, la extensión no estaría logrando el objetivo. Ante esa preocupación, identifican el modelo extensionista alternativo o crítico, cuya particularidad es que propone una visión crítica de la estructura social y promueve la libertad de las clases populares como constructoras de su propia historia y realidad. El modelo alternativo o crítico reúne una gama diversa de prácticas desarrolladas en contextos latinoamericanos bajo una visión crítica, histórica, relacional y compleja de sus estructuras sociales (Monge et al, 2020). 
Por otro lado, al modelo extensionista alternativo o critico se le atribuyen muchas de las propuestas de Paulo Freire (1984), entre ellas el planteamiento de conseguir reinventar la sociedad. Dicho de otro modo, la extensión pide desarrollar procesos de encuentro que permitan una escucha permanente, con diversos espacios de diálogo, problematización, reflexión y crítica popular. Además, tal acto demanda la comprensión mutua, al otro u otra, y la construcción conjunta por esa libertad e indagación de orientaciones hacia una sociedad más justa y equitativa. Por ello, para Freire fue clave no sólo el hecho de que las clases populares llegaran al poder, sino que éstas pudieran reinventarlo mediante una dimensión dialógica, pero crítica, abierta a la participación cada vez más profunda, capaz de romper el binomio dominador-dominado.

A partir de las aportaciones de Freire, continuadas por Tommasino et al. (2006), varias universidades de Centro y Sudamérica permanecen en la búsqueda constante de una extensión universitaria que permita ese diálogo crítico de saberes. Por ejemplo, una de las definiciones más reconocidas en la Universidad Nacional de Costa Rica (UNA), es la siguiente:

Procesos de creación y desarrollo de las capacidades de sus actores sociales, institucionales y locales, y de transformación social integral para una mayor calidad de vida de las comunidades. La extensión nutre la formación integral del académico y del estudiante y fortalece el compromiso que demanda el desarroIlo humano. Comprende acciones conjuntas sociedad-universidad, continuas y planificadas, para la generación de una mejora social integral y la retroalimentación del quehacer universitario (UNA, 2009, p. 6).

Las definiciones de extensión universitaria -desde la versatilidad de sus enfoques-, evoca la interacción entre universidad y sociedad, aunque arrastrados desde su historia permanezcan hasta los actuales días el eventismo, el estructuralismo y el reduccionismo, tan incidentes en su calidad. Una consecuencia de lo anterior es la palidez de su integración con los restantes procesos, convertida en un reclamo sistemático en la actualidad.

Es importante destacar otros contextos, como el cubano, donde la presunción de la relación didáctica entre el carácter de función y proceso de la extensión ha motivado a desarrollar propuestas sustentadas por el modelo 
integral de Valsagna (1997), posicionamiento que no disiente el modelo crítico (Monge et al, 2020). Así, se propon la promoción de cultura con orientaciones sociales específicas, a partir de necesidades sentidas, en respuesta a los principios de diálogo, interactividad, contextuación y creatividad (Monge et al, 2020). Para tales efectos, los procesos universitarios promueven el desarrollo de la sociedad desde la participación de las personas, como agentes de su propio desarrollo

El cumplimiento de este encargo social no se corresponde, como muchos autores lo atribuyen, a una función específica de la universidad (Extensión), sino a la institución en su conjunto. No es únicamente la extensión el factor de cambio, ni se puede considerar como la única expresión del vínculo Universidad-Sociedad, el que requiere de la participación e integración de las funciones universitarias sustantivas (Docencia, Investigación y Extensión) (González 1996, p. 39).

\section{La extensión universitaria como función y proceso}

La definición de extensión como proceso y función fue aportada por González (1996), sustentada en la comprensión de la extensión entendida como

Sistema de interacciones de la Universidad y la Sociedad, mediante la actividad y la comunicación, que se realizan dentro y fuera del centro de educación superior, con el propósito de promover [el subrayado pertenece al texto original] la cultura en la comunidad universitaria y extrauniversitaria, para contribuir a su desarrollo cultural (p. 43).

De acuerdo con el mismo autor, la definición de la extensión como proceso refiere a aquella parte del sistema de interacciones entre sujetos participantes (universidad ysociedad) cuya finalidad busca promover la cultura, sea con comunidades intra o extrauniversitarias. La comunidad es comprendida como aquel conjunto de personas reunidas por intereses comunes y no bajo concepciones geográficas, por ello se da la relación entre enfoques y sujetos sin que signifique que la comunidad esté delimitada geográficamente. El carácter de proceso de la extensión es "una manifestación de la ley didáctica de la relación entre la Universidad y la Sociedad, ley que se establece a través de la función extensionista, que constituye su objeto" (González, 1996, p. 43). Por ello, como proceso atiende el 
cumplimiento de leyes vinculadas a las relaciones externas del proceso con el medio y de nexos internos del proceso.

La extensión, al ser un proceso, da cuenta de varios componentes constitutivos que van desde la definición de problemas socioculturales, basados en intereses generados por las necesidades sentidas, el objeto, el objetivo, su contenido, la metodología, los medios y formas; hasta las acciones de evaluación que intervienen durante las interrelaciones de la universidad con sujetos (o comunidades) externos e internos (González, 1996). Dado su carácter de proceso universitario, su desarrollo muestra etapas definidas con antelación, formuladas rigurosamente, éticamente orientadas y progresivas, con fines debidamente establecidos y gestionados, en igualdad a la investigación y la docencia. Por tanto, se rechaza la improvisación y el mero activismo (Monge et al, 2020). Es un proceso cuyo desarrollo permite resultados de cambios o transformaciones sociales, generalmente conseguidos a largo plazo. De ahí que el autor afirme que:

[L]a extensión universitaria como función expresa las características externas [necesidades sociales], fenoménicas; como proceso, explica sus propiedades esenciales y determina las leyes de su comportamiento [a veces en términos de programas, proyectos y actividades académicos y otras en cursos o prácticas contenidas en los planes de estudios de grado] (González, 1996, p. 52).

Por la naturaleza extensionista, los colectivos externos son los más frecuentes, los cuales, privilegian la satisfacción de intereses comunes mediante soluciones sostenibles que atienden las necesidades de grupos vulnerabilizados respecto a la comunicación entre universidad y empresa (Monge et al, 2020; González, 1996). Por eso, la función de la extensión comprende lo externo, lo visible, lo fenoménico y declara ocho funciones generales de la extensión universitaria (González, 1996). Esas ocho funciones fueron resumidas por Monge, González y Méndez (2020) en: (a) formación profesional del estudiantado, (b) acciones de superación profesional para los graduados universitarios y de la población en general, (c) difusión de los resultados científicos, (d) programación variada y sistemática de opciones culturales y recreativas, (e) agenda de diferentes expresiones de la cultura; (f), atención al sistema de la cultura física y el deporte, (g) fortalecimiento sistemático de las instituciones culturales universitarias y,(h) divulgación de los resultados de la educación superior. Los 
autores concuerdan en la existencia de una relación didáctica compleja entre el proceso y la función extensionista y un vínculo estrecho y sólido entre la extensión y el objetivo de promover cultura. Por ello, la metodología es la promoción cultural tanto para el proceso como para la función, entendida como "conjunto de acciones que impulsan el desarrollo del ciclo reproductivo de la cultura" (González, 1996, p. 46). El carácter inclusivo sin favorecer a élites fue adicionado a esa promoción cultural universitaria en otras investigaciones que integraron el criterio de Zea

En nuestros días y según con los poderosos medios de información y difusión con los que se cuenta, se viene hablando con insistencia de dos clases de cultura, la cultura de masas o popular y la cultura de élite. Y en relación con ellas dos tipos de difusión, la que se considera puede estar al alcance de las masas (...) y la que solo podría estar a la altura de la comprensión de élites especializadas (Zea, 1981 citado por González, 2006, p. 21).

De esta manera, se definió la promoción cultural universitaria como:

[Una] pedagogía de colaboración, igualdad, donde se reconoce la autonomía del individuo en la medida que participa en el desarrollo de la comunidad y de la sociedad en general; refuta la transmisión con la apropiación contextuada de la cultura, bajo los principios de asequibilidad y acceso, tiene en cuenta la diversidad de modo que se extienda la vida científica de la universidad a la cotidiana de la comunidad en una perspectiva de ampliación de la conciencia humana (González, 2006, p. 33).

Ante lo expuesto, González (2006) pone énfasis en el sentido amplio que adquiere el término cultura en relación con la promoción cultural universitaria al confrontar, especialmente concepciones que la acotan al arte, el deporte o el economicismo. Por ello, la define como:

Cultura de la nueva universidad (...) [es un] proceso equitativo y personológico, dialógico e interactivo, creativo y contextualizado de apropiación del capital cultural, en condiciones de colaboración, que contribuya, en el plano individual, a mejorar la calidad de vida de los sujetos y en el más universal, al progreso social 
para la sostenibilidad patrimonial y ecológica local, nacional, regional y del planeta (pp. 20-21).

A la luz de lo expuesto, la extensión se comprende como la función académica universitaria que permite a la universidad una relación de forma totalizadora, sistemática y dialéctica con la comunidad nacional. Es un proceso porque contiene una serie de etapas desde su concepción de la problemática social sentida, la formulación y los fines establecidos y formalizados adecuadamente, de la mano con la investigación y la docencia. Para abarcar la mayor integralidad posible de actores y disciplinas, se orienta al tratamiento inter y transdisciplinario con la participación activa del personal docente y sector estudiantil, quienes trabajan horizontal y dialógicamente con los colectivos sociales y ciudadanía interna o externa de la universidad. Esta acción permite una adecuada articulación entre el conocimiento científico y los saberes populares de manera que se combinan, complementan e integran entre sí para dar origen a un conocimiento nuevo producido con el diálogo en colectividad.

De las definiciones de extensión, la que la distingue como función y proceso desde una perspectiva integracionista, permite mayor acercamiento al cómo lograr la manifestación de su naturaleza totalizadora. Es la integración de los procesos universitarios presentes en la extensión como núcleo básico que le da a esta el título de vía expedita en el cumplimiento de la misión extrínseca de la universidad que se registra en la literatura especializada (González, 2006).

La extensión se comprende como la función (lo externo fenomenológico) y proceso (lo interno: formación profesional y transformación social) académico universitario que permite a la universidad una relación de forma totalizadora, sistemática y dialéctica con la comunidad nacional e internacional. Es un proceso de contenido inter y transdisciplinario mediante el cual se da la participación activa del personal docente y sector estudiantil, quienes se integran a trabajar con los colectivos sociales de diferentes comunidades mediante una adecuada articulación de los conocimientos y saberes de ambas partes. Tal acción apunta a la transformación de la realidad social, lo que según varios autores, se caracteriza por su complejidad, diversidad, dinámica y cambio (Montañez, 2015; Morín, 2001). Ante lo que surgen cuestionamientos tales como: ¿De dónde partimos conceptualmente para abordar la realidad social en tiempos de incertidumbre? 
¿cómo se conceptualiza sociedad, entorno y comunidad y su relación?, ¿qué importancia tiene la otredad en la interacción y contextuación universidad-sociedad?

\section{Realidad social}

En un mundo en el que la vida pasa muy rápido, con preocupaciones diversas, en la mayoría de las ocasiones se olvida considerar el tiempo y espacio en la reflexión de aspectos claves del vivir, como son la naturaleza de la humanidad, las relaciones, las emociones y las fuerzas espirituales. Esta situación afecta los avances fructíferos del conocimiento. Ante esto, Wallerstein (2006) aporta la identificación de tres problemas fundamentales para la incorporación de estas variables. El primero atiende la relación entre el investigador y la investigación, a lo cual refiere los señalamientos de Weber sobre la trayectoria del pensamiento moderno como el "desencantamiento del mundo" (p. 81). Por otra parte, Wallerstein (2006), apunta a lo indicado por Prigogine y Stengers (1986), sobre conseguir un reencantamiento del mundo entendido como "aquel llamado a derribar las barreras artificiales entre los seres humanos y la naturaleza, a reconocer que ambas forman parte de un universo único enmarcado por la flecha del tiempo" (p. 81). En segundo lugar, se cuestiona cómo reinsertar el tiempo y el espacio como variables de análisis, no vistas como fenómenos arbitrarios, sino como parte de la construcción social. Y tercero, anota el problema de cómo superar las separaciones entre lo político, lo económico y lo social.

A la problemática expuesta, Wallerstein (2004) y Houtart (2009), adicionan la incertidumbre que, desde el inicio de la historia, ha inspirado temor y sensación de peligro y se ha incrementado en los últimos treinta años. A ella se le atribuye la generación de un periodo de transición y, por ende, un futuro incierto en el que la realidad obliga a reflexionar en torno a la probabilidad en lugar de la certeza y la tendencia de alejarse del equilibrio, la bifurcación y la flecha del tiempo (Wallerstein, 2004). Coinciden en que la incertidumbre no sugiere "la atomización de la realidad, la ausencia de estructuras, sino aceptar que la realidad no es lineal, ni determinada, que existe una multiplicidad de factores en interacción y afirmar el lugar formal de lo cualitativo" (Houtart, 2009, p. 24).

La incertidumbre surge en una realidad dinámica, cambiante, y hoy más que nunca se aleja de lo que se considera certero y de todo aquello que se 
pueda predecir fácilmente. Al no existir una realidad única, de la cual, las personas son parte de ella, el ser humano no puede trascender la realidad. Montañez (2015), comprende que en esa dinámica que tiende al cambio, el otro u otra no deberían considerarse inertes, por el contrario, es preciso tener claridad que el otro al actuar, transforma su realidad y, por ende, la de quienes lo rodean, tomando relevancia el papel del otro. Ante estos postulados, se requiere que las diferentes ciencias perciban la realidad no como algo estático, sino como lo propone Morin (2005, citado en Houtart, 2009), desde un orden, desorden, interacción y organizaciónal cual le denomina el paradigma de la auto-eco-re-organización.

Esta consideración dialéctica en la dinámica de cambio, entalpía y entropía de la realidad social, obliga y compromete a asumir una definición de extensión que por analogía responda a tal concepto de realidad para bien social, nacional y del planeta; quedando evidente el divorcio entre la concepción de realidad social expuesta y los modelos tradicionalistas y economicistas de extensión antes dicho.

Pero, aún queda por analizar qué entender como sociedad, esa con la que la universidad se comunica usando como vía a la extensión ¿Qué significa entorno cuando se refiere la extensión a su principio de contextualización? $\mathrm{y}$, por último, ¿qué asumir conceptualmente como comunidad? esa célula básica de operaciones reales donde se constata el impacto de la universidad mediante proyectos extensionistas o comunitarios, con evidencias como avales de las transformaciones sociales acontecidas mediante un proceso de acompañamiento entre universidad ycomunidad.

\section{Sociedad, entorno y comunidad}

El objetivo del presente texto puede generar en el lector la expectativa de que las categorías que se relacionan con la concepción de extensión universitaria sean tratadas por separado. Sin embargo, la profunda dialéctica de las categorías sociedad,sntorno ycomunidad inspira a la autora a exponerlas en el vínculo que estas poseen, sobre todo en relación al proceso que representa la vía expedita de comunicación entre universidad y sociedad.

La sociedad, según Durkheim (1986), es más que la suma de los individuos, pues hay otros elementos que también la integran, aunque son 
los individuos los únicos integrantes activos. Por ello, se considera que la sociedad no se compone de personas, más bien, estas forman parte del entorno de la sociedad como sistema. Por lo tanto, se establece que para entender la realidad social se debe mirarla como sistema, para dejar claro el carácter socio-constructivista del mismo. Los sistemas, a su vez, presentan varias características, de acuerdo con Orozco y Gaviria (2012), todos los sistemas son mediadores de la complejidad, tienen la capacidad de selección entre alternativas y están ordenados para el cumplimiento de una determinada función. Es el sistema el que propicia unidad al entorno, que en criterio de Luhmann (1973, citado en: Arnold \& Rodríguez,1991), es lo que da existencia a lo demás. De ahí, la anterior afirmación de que el entorno tiene su unidad a partir del sistema, puesto que cada uno de los sistemas tiene su propio entorno.

De lo anterior se infiere que el entorno es más complejo que el sistema, así que la complejidad del sistema consiste en que se constituye de subsistemas, con sus respectivos elementos. El sistema presenta características, posibilidades y probabilidades.

Al considerar las interacciones en lo interno de cada sistema, entre sistemas y con el entorno (realidad) como un todo, lo que es aplicable al proceso extensionista en su relación sistemática con un entorno cambiante de constantes demandas. Por ejemplo, en una determinada comunidad se puede observar que el entorno está compuesto por una serie de sistemas: el educativo, económico, ambiental, social, organizacional, entre otros. Cada sistema a su vez tiene una serie de elementos, los cuales tienen su propia historia, que en algunas ocasiones se quieren cambiar para tener mejor calidad de vida. Es muy difícil, casi irracional, pensar que se pueden cambiar todos los sistemas a la misma vez, aunque exista comunicación entre uno y el otro, la dinámica de la realidad lo dificulta, porque quienes la conforman no son elementos mecánicos fáciles de cambiar, sino que son elementos complejos: sujetos activos con capacidad para tomar decisiones, vinculados a un contexto histórico y cultural insoslayable para quienes quieran transformar su realidad, ya sea desde lo individual o institucional como es el caso de la extensión.

En este contexto, ya sea de manera individual o colectiva, el ser humano interactúa, y no lejos de ello, se encuentra el tejido organizacional e institucional que busca analizar los procesos sociales. Un ejemplo de este tipo 
de instituciones son las universidades alrededor del mundo, las cuales, buscan interacción social a la vez que son demandadas por la sociedad en la solución de las necesidades sentidas de sus comunidades.

Las comunidades constitutivas de la sociedad deben ser entendidas como un grupo de sujetos reunidos por intereses semejantes, no precisamente territoriales. Es una identidad común mediante la diferenciación de otros grupos o comunidades (generalmente por signos o acciones), la cual es compartida, elaborada y socializada entre sus integrantes. Generalmente, una comunidad se une bajo la necesidad o mejora de un objetivo afín, como puede ser el bien común, aunque esto no es algo necesario, puesto que basta con una identidad grupal para conformar una comunidad sin la necesidad de un objetivo específico. La comunidad no es estática, es movimiento, sufre crisis, se reorganiza, hay épocas de resistencia y de búsqueda de alternativas comunes localmente y de manera autónoma. Sus procesos socioambientales, socioculturales, socioeconómicos son singularidades que las distinguen a unas comunidades de otras (Torres, 2013).

También en la comunidad llegan a presentarse expresiones de procesos más amplios como las formas globales dominantes del consumo y desecho generalizadas por la modernidad, las cuales trastocan su integridad vital en su salud, su cultura, la economía y en los ecosistemas. En todo este proceso juega un gran papel la universidad como célula cultural de la sociedad (Núñez, 1999), predominante desde la extensión y su misión de promover cultura.

Como lo señala D’Angelo (2004), los patrones de interacción social se producen en el entrecruzamiento de representaciones, ansiedades, expectativas y, más ampliamente, en las configuraciones de la subjetividad social. Esas configuraciones conectan una gran diversidad de variables como género, edad, posición socioeconómica, raza, pautas de relación familiar y roles sociales, que expresan las dimensiones de saber, deseo, poder y discurso de los sujetos actuantes. Por esa razón, se considera fundamental utilizar diferentes "lentillas" al mirar ya que existe una dualidad de pensamiento en un mismo ser: individual y sociocolectiva.

La extensión universitaria, desde la visión de que el conocimiento es una construcción social, requiere el reconocimiento al otro u otra (otredad), previo a cualquier proceso que implique el análisis y la interacción con la 
realidad. Desde esa interconexión existente, el individuo está en la sociedad y, esta, en el individuo, por tanto, cabe cuestionar ¿sobre qué paradigma tendría que orientar la universidad ese cambio y transformación social?

\section{Paradigmas de investigación en contribución a la extensión universitaria}

Por ser tan cierto que la extensión es el acercamiento a la realidad social, mediante diálogo de saberes para resolver necesidades sentidas de una comunidad dada, es claro que este proceso está estrechamente relacionado con la investigación, por lo que, a la hora de desarrollar tales saberes es importante posicionarse en un paradigma determinado. Esta relación con la sociedad se somete a determinadas posturas de visión de mundo que poseen los individuos que participan en el diálogo. En tal proceso se integra un sistema de declaraciones y creencias sobre la realidad, los individuos, el lugar que ocupan ellos y sus relaciones sobre lo existente (Flores, 2004 citado en Ramos, 2015).

Ramos, hace referencia a que un paradigma engloba un sistema de creencias sobre la realidad, la visión del mundo, el lugar que el individuo ocupa en él y las diversas relaciones que esa postura permitiría con lo que se considera existente. Por eso, coincide en que el paradigma constituye los lentes con los que una persona observa la realidad y determina la manera con que mira e interpreta. Por lo tanto, tener claridad del paradigma desde el que se está mirando permite una postura ética y política para el acercamiento a la misma realidad. Es así como, cobra sentido el paradigma en la extensión, tanto para quien lo protagoniza como para quienes forman parte indirecta del proceso extensionista. Por ejemplo, Ramos (2015) identifica cuatro tipos de paradigmas:

1. El positivismo: las ciencias exactas son el origen de este paradigma. Establece que la realidad es absoluta y aprehensible por el ser humano, además, es regida por las leyes y mecanismos naturales.

2. El post- positivismo: indica que la realidad existe, pero que solo puede ser entendida parcialmente. Reconoce desde lo ontológico que la realidad no puede ser comprendida totalmente ya que las herramientas utilizadas para observarla y analizarla no son perfectas así como el ser humano. 
3. El constructivismo: dicho paradigma promueve la idea de que el saber del mundo se construye por el interaccionismo social, es decir, desde lo ontológico no se intenta controlar o predecir la realidad sino reconstruirla en la medida que exista en la mente de los constructores. Este paradigma afirma que no existen realidades únicas sino construcciones de acuerdo a las percepciones de cada individuo.

4. La teoría crítica: va más allá de la reflexión. Establece que quien investiga o se acerca a la realidad busca generar cambios y la liberación de un determinado grupo o contexto. Se caracteriza por su visión holística y dialéctica. Todos los que participan están comprometidos con el cambio social; comprensión social de necesidades e intereses y una transformación de las estructuras sociales, basada en la liberación de los individuos que conforman el contexto social. Este paradigma se diferencia de los demás al proponer no sólo observar y pensar, sino actuar. $Y$ desde la perspectiva ontológica considera que la realidad es fruto de la influencia de elementos sociales, políticos, culturales, económicos, entre otros.

\section{Relación de la extensión crítica con los paradigmas interpretativo y crítico}

Al analizar los diferentes paradigmas se considera que la extensión universitaria desde la perspectiva crítica se fundamenta en el paradigma teórico crítico, por cuanto la interacción que se tiene como extensionista con las comunidades busca conocer la realidad y adquirir una visión de esta, al ejecutar un proceso participativo y contextualizado de transformación desde los sectores sociales con los que se trabaja.

La extensión crítica promueve el diálogo y la reciprocidad social, al partir de la identificación de las necesidades en conjunto con la población, reflexionar acerca de estas y actuar sobre las mismas en busca de soluciones, por medio de la articulación de esfuerzos de los diferentes participantes y por la coordinación con las instituciones.

En los procesos de la extensión universitaria se busca que, en el desarrollo de cada una de las fases del proceso, la población (grupo determinado de la comunidad) participe activamente con el objetivo de que realmente se dé un diálogo de saberes entre el equipo extensionista que tiene un 
conocimiento técnico y las personas de la comunidad quienes tienen un conocimiento vivencial.

Desde la extensión crítica se considera de suma importancia para analizar la realidad comprender cuatro dimensiones claves, la sociocultural, ambiental, económica y político- institucional, ya que, como antes quedó expuesto, la realidad está conformada por una serie de aspectos que la hacen ser diversa, dinámica y compleja.

La extensión crítica promueve que la población concientice que muchas de las necesidades que se consideran individuales, son colectivas. Además, al socializar las problemáticas del otro u otra, se reconozcan como propias, lo que posibilita la unión para buscar soluciones alternativas en conjunto a las diferentes necesidades.

Es así como, lejos de considerar la extensión como una simple área de la universidad ocupada de su proyección a la sociedad, se concibe como aquel proceso que permite la retroalimentación bidireccional entre universidad y sociedad. La reflexión con las comunidades en diferentes contextos permite a la universidad orientar la docencia, lo que hace cumplir el principio pedagógico de la unidad de la práctica con la teoría desde el reconocimiento de las necesidades sentidas de las comunidades y las de formación profesional del estudiante de modo concreto.

\section{El cambio social en la realidad}

El cambio se define como "una sucesión de diferencias en el tiempo en una identidad persistente" (Nisbet, 1975, p. 294), de esta forma se puede decir que los cambios se visualizan cuando se tiene una identidad y se manifiestan diferencias en el tiempo. Un ejemplo de ello son los cambios que ha tenido la religión en un lapso temporal, han pasado muchos siglos para observar algunos cambios.

Otra característica del cambio es que no puede deducirse o derivarse empíricamente de los elementos de la estructura social, es decir, no se puede limitar a lo social ni solo a lo biológico, ya que son diversos los elementos referidos al tiempo, las circunstancias y el lugar. 
Por lo tanto, el cambio no es un rasgo constante de la vida social, ya que no toda interacción genera un cambio.Este requiere de persistencia y, en la mayoría de los casos, de crisis. De esta última en la medida en que se "concibe como la relación entre el ser humano y el ambiente, precipitada por la incapacidad del ser humano de seguir por más tiempo con determinado modo de comportamiento" (Nisbet, 1975, p. 307). En el momento que se da esa ruptura y se genera la crisis, permite con frecuencia los cambios aunque en la mayoría de las veces los seres humanos se resisten a ello.

Así como el cambio está relacionado con la crisis lo está con el acontecimiento, el cuál según su magnitud o significado puede generar cambios en la vida de una persona e incluso de una nación.

Nisbet (1975) sintetiza algunas razones por las que se puede afirmar que los cambios sociales son necesarios. Primero, muy pocas formas de comportamiento de la sociedad pueden ser continuas, es decir, el cambio es necesario por la misma naturaleza del comportamiento social. Por ello, es clave entender la naturaleza del vínculo social, para comprender las estructuras sociales que se han modificado en una situación de crisis. En la mayoría de los casos, no se busca el cambio en algunos comportamientos como la religión, el nacionalismo político u otros. Cuando se generan cambios es porque en definitiva se hace imposible mantener la continuidad de un comportamiento.

Ante este análisis se puede decir que los cambios no se dan de la noche a la mañana, al menos que suceda un acontecimiento fuera de control que los genere. Mas bien, es lento y está relacionado con muchos elementos del entorno, desde lo individual hasta las relaciones sociales. Se apunta a los cambios como el sentido en que se procesa la experiencia y la acción, cuya estructura adquiere forma totalizante $\mathrm{o}$ en dimensiones especializadas.

Por otra parte, Weber (1984) señaló que la acción social debe entenderse como una conducta humana, siempre que el sujeto o los sujetos enlacen en ella un sentido subjetivo. Como no toda relación es una acción social, Weber la caracterizó. Esta acción puede ser de varios tipos, sea de carácter racional con arreglo a fines, racional con arreglo a valores, afectiva o tradicional. Un tipo no excluye al otro, por el contrario, se pueden complementar. 
Para la acción social es fundamental entender el entorno, es decir, la realidad de una comunidad o un determinado grupo. En el caso de la extensión universitaria es evidente que quienes la conocen a fondo son las personas que viven en ella. Consecuentemente, toma fuerza la participación de agentes del contexto en el proceso extensionista, en los diferentes procesos sociales y comunitarios.

Gonzáles y Duke (1990) explican la extensión universitaria como una forma de intervención social capaz de permitir a los participantes auto reconocerse como actores protagónicos, que mientras comparten situaciones determinadas desde sus intereses, expectativas y necesidades comunes, consiguen ampliar sus oportunidades de identificación individual y colectiva. Se esperaría que, mediante la participación activa, los individuos logren la capacidad de generar agencia o actuación autónoma colectiva frente a los desafíos socio ambientales y a los otros actores sociales y políticos. $Y$ es que los espacios de encuentro comunitario deben ser aquellos que permitan a los actores sociales no sólo tratar de comprender su realidad (análisis de necesidades y potencialidades), sino tratar de explicarla en conjunto, al sentirse capaces en el proceso de actuar sobre su realidad de una forma organizada, al entender que lo que le suceda al otro u otra no solo altera su entorno, sino el de todos y que por tanto, la colectividad, el compromiso y el apoyo mutuo son importantes en los procesos comunitarios y de transformación social.

Al cierre del análisis sobre la definición de extensión y demás categorías implicadas, la extensión se asume desde una cosmovisión que determina el posicionamiento de quienes la ejecutan respecto a las categorías que involucra, unas tratadas en el texto; y otras, posibles. Resulta también fundamental la dimensión axiológica y comunicacional de la universidad ante la sociedad a la que se debe. Reflexionar desde la visión del modelo crítico e integrador de extensión implica un compromiso sociopolítico de la universidad de hacer realidad el mejoramiento profesional y humano de sus estudiantes y el ideario altruista ante la sociedad de la nación, a la que responde la universidad. 


\section{Agradecimiento}

Se agradece profundamente a la PhD. Maritza González-Moreno, de la Universidad de la Habana, Cuba, por sus rigorosas observaciones y cuestionamientos ofrecidos para este documento.

\section{Referencias}

Arnold, M. \& Rodríguez, D. (1991). Teoría de los sistemas sociales: las bases de la perspectiva Luhmanniana. Revista de Estudios Sociales (69) 93-110.

Bosco, P. (1973). Extensión o educación: una disyuntiva crítica. Desarrollo Rural en las Américas, 5 (3) 166-175.

D’ Angelo, O. (2004). La subjetividad y la complejidad. Procesos de construcción y transformación individual y social. Consejo Latinoamericano de las Ciencias Sociales.

Durkheim, E. (1986). Las reglas del método sociológico. México: Fondo de Cultura Económica.

Freire, P. (1965). La educación como práctica de la libertad. Argentina Editores.

Freire, P. (1984). ¿Extensión o comunicación?: la concientización en el medio rural. Siglo XXI.

Ghiso, A. (2000). Potenciando la diversidad (diálogo de saberes, una práctica hermenéutica colectiva). Aportes, 53, 58-71.

Gonzáles, E. \& Duque P. (1990). La elección de juntas Administrativa locales de Cali. Revista Foro, 12, 77-88.

González, F. (2002). Un modelo de gestión de la extensión universitaria para la Universidad de Pinar del Río. Centro de Estudios de Ciencias de la Educación Superior. http://rc.upr.edu.cu/bitstream/DICT/1744/1/ Mercedes\%20Gonz\%C3\%A1lez\%20Fern\%C3\%A1ndez-Larrea.pdf 
González, M. (2006). Formulación teórico-metodológica de la promoción cultural de la investigación para la integración de los procesos universitarios de extensión e investigación en el Instituto Superior Politécnico José Antonio Echeverría. [Tesis]. Centro de Referencia para la Educación de Avanzada.

González, M. (2013). Concepción de cultura para el modelo integral de extensión universitaria. Revista Referencia Pedagógica, 1 (2), 104115. http://rrp.cujae.edu.cu/index.php/rrp/article/download/25/33 30-01-2019

González, R. (1996). Un modelo de extensión universitaria para la extensión universitaria. Su aplicación a la Cultura Física y el Deporte. [Tesis]. Instituto Superior de Cultura Física "Manuel Fajardo".

Houtart, F. (2009). La ética de la incertidumbre en las ciencias sociales. Consejo Latinoamericano de las Ciencias Sociales y Ruth Casa Editorial.

Mollis, M. (2003). Las Universidades en América Latina ¿Reformadas o alteradas? La cosmética del poder financiero. Consejo Latinoamericano de Ciencias Sociales.

Monge, C. (2020). La universidad latinoamericana en la sociedad. Análisis de la relación entre universidad y comunidad desde el enfoque de capacidades para el desarrollo humano. [Tesis]. Universitat Politècnica de València.

Monge, C., González M. \& Méndez, N. (2020). De la Reforma de Córdoba a la extensión crítica: un breve recorrido por la extensión universitaria latinoamericana. Editorial Letra Maya.

Montañez, J. (2015). El otro como estructura disipativa. Universidad de Costa Rica.

Morin, E. (2001). Introducción al Pensamiento Complejo. Gedisa.

Nisbet, R. (1975). Introducción a la sociología. El vínculo social. Editorial Vicens-Vives. 
Núñez, C. (2006). Sentir, amar, pensar... la senda de la tierra. V Encuentro Internacional sendas de Freire opresiones, resistencias y emancipaciones en un nuevo paradigma de vida. Instituto Paulo Freire de España y Crec.

Núñez, J. (1999). La ciencia y la tecnología como procesos sociales. Lo que la educación científica no debería olvidar. Editorial Félix Varela.

Orozco, D. \& Gaviria, A (2012). Una mirada teórica y metodológica a la obra de Niklas Luhmann: entre sistema y entorno. Revista Colombiana de Ciencias Sociales 3 (1) 133-146

Ramos, C. (2015). Los paradigmas de la investigación científica. Avances en psicología 23 (1) 9-17.

Sánchez, V. (1989). Concepción y Políticas de Extensión. Vicerrectoría de Extensión, Universidad Nacional.

Tommasino, H. \& Cano, A. (2016). Modelos de extensión universitaria en las universidades latinoamericanas en el siglo XXI: tendencias y controversias. Universidades (67) 7-24.

Tommasino, H., González, M., Guedes, E. \& Prieto, M. (2006). Extensión Critica: los Aportes de Paulo Freire. En H. Tommasino; P. De Hegedüs. (Eds). Extensión: Reflexión para la intervención en el medio urbano y rural. (pp. 121-135). Universidad de la República, Uruguay.

Torres, A. (2013). El retorno a la comunidad. Problemas, debates y desafíos de vivir juntos. Fundación Centro Internacional de Educación y Desarrollo Humano y Editorial El Búho.

Tünnermann, C. (1981). Ensayos sobre la universidad latinoamericana. EDUCA.

Tünnermann, C. (2000). El nuevo concepto de extensión universitaria y difusión cultural y su relación con las políticas de desarrollo cultural en América Latina. Anuario de Estudios. 
Universidad Nacional. (15 de noviembre de 2009). Lineamientos para la gestión de programas, proyectos y actividades. Gaceta N. ${ }^{a}$ 17-2009. https://documentos.una.ac.cr/handle/unadocs/1793

Valsagna, V. (1997). Extensión y comunicación. Un enfoque comunicacional de la extensión universitaria". [Ponencia]. II Encuentro Latinoamericano de Extensión Universitaria y I Congreso Nacional de Extensión de la Educación Superior.

Wallerstein, I. (2004). Las Incertidumbres de/ Saber. Editorial Gedisa.

Wallerstein, I. (2006). Abrir las Ciencias Sociales. Editorial Siglo XXI.

Weber, M. (1984). Economía y Sociedad. Fondo Cultura Económica. Decimo-sétima Editorial. 
